\title{
ANC experiments for nuclear astrophysics in NPI CAS
}

\author{
J. Mrazek ${ }^{1, \star}$, V. Burjan ${ }^{1}$, V. Kroha ${ }^{1}$, A.M.Mukhamedzhanov ${ }^{3}$, R. Tribble ${ }^{3}$, C. Spitaleri ${ }^{2}$ I. Siváček ${ }^{1}$,

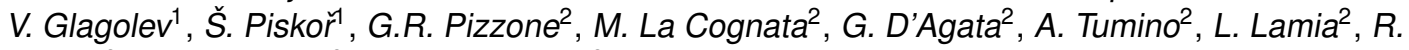 \\ Sparta $^{2}$, G. Rapisarda ${ }^{2}$, and S. Romano ${ }^{2}$ \\ ${ }^{1}$ Nuclear Physics Institute of the Czech Academy of Sciences, p.r.i., Řež 130, 25068 Řež, Czech Republic \\ ${ }^{2}$ Instituto Nazionale di Fisica Nucleare, LNS, Via S. Sofia 62, 95125 Catania CT, Italy \\ ${ }^{3}$ Cyclotron Institute, Texas A\&M University, College Station, Texas 77842
}

\begin{abstract}
Asymptotic Normalization Coefficients method is one of the indirect methods used in nuclear astrophysics. The method allows to deduce a direct part of a radiative capture cross section from the measurement of a two body reaction under specific conditions. The experimental works started in collaboration of NPI CAS, TAMU and INFN LNS from 90-ties and continue till the present. The introduction to this method is presented and experimental experiences from NPI CAS and the collaboration are shared.
\end{abstract}

\section{Introduction}

Nuclear reactions at low energies are of a specific interest in astrophysics. A number of scenarios of stellar nucleosynthesis involve low energy reactions, frequently radiative capture and these reaction frequently - due to the low energy and other conditions proceed peripherally. However, direct measurement of these reactions is difficult and technological development makes it more and more accessible only in the last decades.

Indirect methods can provide the information that is currently not possible to obtain directly, but also can bring another piece of information to the direct measurements, that is necessary to draw proper conclusions.

Asymptotic Normalization Coefficients method can extract information about direct radiative capture like $(p, \gamma),(n, \gamma),(\alpha, \gamma)$ at low energies. There is also one auxiliary advantage for older accelerator devices, who were not modernized or decommissioned in the last decades. The older accelerator types can be used with indirect methods, if they can accelerate light ion beams like deuteron and ${ }^{3} \mathrm{He}$ at energies around $20 \mathrm{MeV}$, which is also a case of the NPI CAS cyclotron U120M. The ANC method measurements were performed here from the dawn of the method from 90-ties until now in collaboration NPI - TA\&MU - INFN-LNS developments driven by A.M.Mukhamedzhanov, R.Tribble and V.Kroha.

\subsection{Historical excursion}

When nuclear reactions started to be studied, Fermi [1] discovered in 1934 an enhancement (resonant) of the cross sections. Soon Breit and Wigner [2] presented their theory of resonances and later,

\footnotetext{
^e-mail:mrazek@ujf.cas.cz
} 
the hypothesis of independence was provided by Bohr 1936, where the reaction proceeds through a compound state and the nucleus looses information about the creation channel. Ghoshal et al. [3] have shown a convincing experimental evidence for such a behavior. $\gamma$ and $\beta$ spectroscopy gave the pictures of nuclear structure, and showed an importance of a collective excitations.
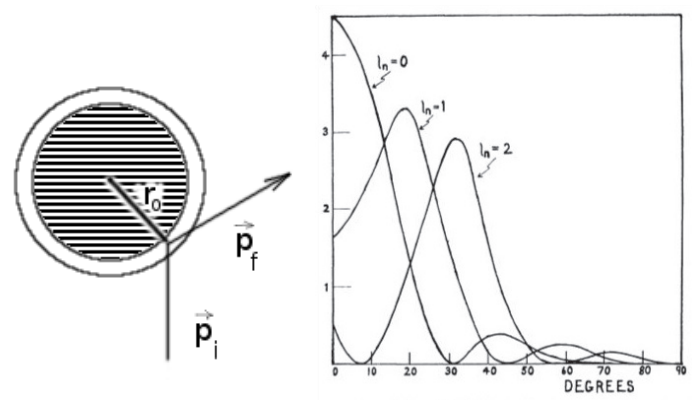

Figure 1. Left panel: S.T.Butler had the idea that the nucleus contains a black (absorption) core and a grazing region, where the direct reactions occurs [4]. Right panel: Angular distributions of direct reactions peak at certain angle that depends on the transferred momentum $L=\left|\overrightarrow{r_{0}} \times\left(\overrightarrow{p_{f}}-\overrightarrow{p_{i}}\right)\right|$. [5]

Single-particle transfer reactions started to be studied in 50-ties and seemed to be they are important for understanding single particle excitations (collective excitations studies were developed with $\beta$ and $\gamma$ spectroscopy). S.T.Butler $[4,5]$ pointed out the validity of the Born approximation and he made a great contribution to direct reactions as a tool for nuclear structure (see Fig.1).

\subsection{Optical model}

A description of a nuclear reaction can be written [6] in a very general way using the the Schrödinger equation $\left(H_{x}+H_{A}+T+\Sigma_{i \in x, j \in A} V_{i j}\right) \Psi_{\alpha}=E \Psi_{\alpha}$ where all nucleons of a projectile $x$ and a target $A$ mutually interact and the index $\alpha$ stands for the input channel or a partition $x+A$.

It is useful to accept some simplifications. The shell model suggests to describe a nucleus as nucleons in a central potential $U$, that represents an average field of all nucleons with corrections that are taken into account as residual interactions $V_{i j}$. By this way, it is intuitive, that the interaction of the approaching projectile and the nucleus is (at large distances) driven by this potential $U$. If there is no exchange of energy or nucleons, then this potential $U$ should solely describe the interaction - the elastic scattering. The Schrödinger equation of the system may be written as $(H+T+U) \Psi=E \Psi$. Then, a direct reaction may be taken as a perturbation of the elastic scattering [6].

Under these considerations, the Schrödinger equation is separable into nuclear coordinates and relative coordinates of the projectile. The solution $\Psi_{\alpha}$ can be written as a product of nuclear $\Phi_{\alpha}$ and relative $\chi_{\alpha}(r)$ wave functions $\Psi_{\alpha}=\Phi_{\alpha} \chi_{\alpha}(r)$, where the corresponding Schrödinger equation may be written $(T+U) \chi_{\alpha}(r)=E \chi_{\alpha}(r)$.

The most traditional shape of the optical potential $U$ is a Woods-Saxon form. To describe the loss of flux due to reactions the potential must also have a complex term and to describe the experimentally observed orbitals it also has an LS term, and frequently a surface term is also introduced.

A transfer reaction has a different output channel, partition $Y+B$ named $\beta$, so it is possible to find another optical potential and a relative wave function $U_{\beta}, \chi_{\beta}(r)$.

\subsection{DWBA approach}

A typical transfer reaction may be depicted as in the diagram in Fig.2. Using the above considerations about the optical potential it is possible to deduce a very important tool in direct nuclear reactions the Distorted-Wave Born Approximation. The details can be found in textbooks e.g. by Glendening 


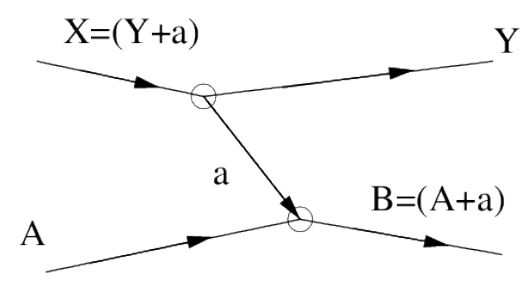

Figure 2. Diagram of a transfer reaction. The vertex contains the information for direct radiative capture.

[6] in chapter 5. This (DWBA) approximation leads to a formula for a transition amplitude between the states before and after the reaction.

The matrix element of the transition between (inelastic) incoming and outgoing channels can be written (in post-form) as

$$
M\left(E_{i}, \theta_{c . m .}\right)=\sum_{M a}\left\langle\chi_{f}^{(-)} I_{A a}^{B}|\Delta V| I_{Y a}^{X} \chi_{i}^{(+)}\right\rangle
$$

where $I_{A a}^{B}$ is the radial overlap function of the system $A+a$ and the nucleus $B, \chi_{i}$ and $\chi_{f}$ are incoming and outgoing (by the potential $U$ ) distorted waves, $\Delta V$ is the perturbation part of the potential $\Delta V=$ $V_{\beta}-U_{\beta}$, where $V$ is a full (unknown to us) potential and $U$ is the optical potential.

The radial overlap function - in general $I_{a b}^{c}$ can be approximated by (model calculated using e.g. Woods-Saxon potential well) bound state wave functions $\varphi_{n, l_{c}, j_{c}}\left(r_{a b}\right)$ through a factor $S_{a b}$

$$
I_{a b l_{c} j_{c}}^{c}\left(r_{a b}\right)=S_{a b \xi_{c} j_{c}}^{1 / 2} \varphi_{n_{c} l_{c} j_{c}}\left(r_{a b}\right) \text {. }
$$

The factor $S_{a b t_{c} j_{c}}^{1 / 2}$ is called the spectroscopic factor (SF) and it was intensively used to study the occupation of different nuclear orbitals. Nowadays, it is still a very good tool to inspect the nuclear structure, but time has shown that it has certain limitations. The SF's depend on the selection of the optical model $(\mathrm{OM})$ potential and there are in general several ambiguities concerning OM's: i) several different potential families can reproduce the angular distribution data, ii) the cross section scales with the potential radius $r_{0}$, iii) the (Woods-Saxon) depth is usually tuned to reproduce the bound states (well depth prescription).

Another important fact about SFs was discussed recently by several authors [7-9]: SFs rely on internal wave functions, which implies that in fact they are not observables.

Despite these facts, it is possible to face these difficulties by a careful treatment of the analysis as was shown e.g. by X.Liu [10]. He compared the previous works with (d,p) and (p,d) reactions with ${ }^{12} \mathrm{C}$ as a target. He finds differences of factor 5 and by a consistent treatment he could reach $15 \%$ variation of the reevaluated data.

The experimentally measured angular distributions $\frac{d \sigma}{d \Omega}$ can be reproduced by DWBA calculations.

$$
\frac{d \sigma}{d \Omega}=\Sigma_{j_{B} j_{x}} S_{A a l_{B} j_{B}} S_{Y a l_{x} j_{x}} \sigma_{l_{B} j_{B} l_{x} j_{x}}^{D W}
$$

$\frac{d \sigma}{d \Omega}$ and $\sigma_{l_{B} j_{B} l_{x} j_{x}}^{D W}$ are related through a coefficient that basically consists of two SF, one of them is usually (for light particles like $\mathrm{d},{ }^{3} \mathrm{He}$ ) known, so the analysis will offer the spectroscopic factor of the studied state.

\section{Asymptotic Normalization Coefficients}

Mukhamedzhanov and Timofeyuk have pointed out in 1990 [11] that the direct radiative capture cross sections of low energy astrophysical reactions usually contain the same radial overlap integral $\left(I_{a b}^{c}\right)$ as 
the (direct) transfer reactions. Since these direct captures frequently proceed at large distances from the nucleus, it is possible to use asymptotic expressions for the radial overlap functions (and bound state wavefunctions (see eq.2) )

$$
\begin{gathered}
I_{\beta \gamma l_{\alpha} j_{\alpha}}^{\alpha}\left(r_{\beta \gamma}\right) \stackrel{r_{\beta \gamma}>R_{N}}{\longrightarrow} C_{\beta \gamma l_{\alpha} j_{\alpha}}^{\alpha} \frac{W_{-\eta_{\alpha}, l_{\alpha}+1 / 2}\left(2 \kappa_{\beta \gamma} r_{\beta \gamma}\right)}{r_{\beta \gamma}} \\
\varphi_{n_{\alpha} l_{\alpha} j_{\alpha}}\left(r_{\beta \gamma}\right) \stackrel{r_{\beta \gamma}>R_{N}}{\longrightarrow} b_{\beta \gamma l_{\alpha} j_{\alpha}} \frac{W_{-\eta_{\alpha}, l_{\alpha}+1 / 2}\left(2 \kappa_{\beta \gamma} r_{\beta \gamma}\right)}{r_{\beta \gamma}},
\end{gathered}
$$

where $W_{-\eta_{\alpha}, l_{\alpha+1 / 2}}$ is a Whitaker function that has a known asymptotics, $W_{-\eta_{I}, l+\frac{1}{2}}\left(-2 k_{I} r\right) \rightarrow$ $e^{-k_{I} r+\eta_{I} \ln \left(2 k_{I} r\right)}$.

The coeficients $C^{2}$ and $b^{2}$ are called ANC and single particle ANC (SPANC), respectively. If we use the above approximation with the eq.3, we obtain a modified formula for comparison of experimental data with the DWBA model:

$$
\frac{d \sigma}{d \Omega}=\Sigma_{j_{B} j_{x}}\left(C_{A a l_{B} j_{B}}^{B}\right)^{2}\left(C_{Y a l_{x} j_{x}}^{X}\right)^{2} \frac{\sigma_{l_{B} j_{B} l_{x} j_{x}}^{D W B{ }_{1}}}{b_{A a l_{B} j_{B}}^{2} b_{Y a l_{x} j_{x}}^{2}}=\Sigma_{j_{B} j_{x}}\left(C_{A a l_{B} j_{B}}^{B}\right)^{2}\left(C_{Y a l_{x} j_{x}}^{X}\right)^{2} R_{l_{B} j_{B} l_{x} j_{x}}
$$

The traditional method of finding spectroscopic factors turns into finding ANCs (for light projectiles can be $C_{Y a}^{X}$ known) based on DWBA analysis giving the function $R$. At first, it does not seem to be an improvement, but in fact, the ANCs have several advantages over SF as was already mentioned in sec. 1.3.

- for a peripheral reaction, the ANCs have at most a small dependence on the selection of the potential. $R_{l_{B} j_{B} l_{x} j_{x}}$ from eq.5 is nearly independent of $b^{2}$

- ANCs are defined in the nuclear exterior, so they are "observable"

- ANC is related to the Nuclear Vertex Constant (NVC) $G_{\beta \gamma l_{\alpha} j_{\alpha}}^{\alpha}=-e^{i \pi\left[\left(l_{\alpha}+\eta_{\alpha}\right) / 2\right]} \frac{\sqrt{\pi}}{\mu_{\alpha}} C_{\beta \gamma l_{\alpha} j_{\alpha}}^{\alpha}$, the same constant that appears $[12,13]$ in direct radiative capture reaction a+A and where $\sigma \sim G_{A B a}^{2}$.

\subsection{Validity tests}

As it was mentioned above, the validity of extraction of ANCs from transfer reactions depends on the peripherality of the transfer reaction. Therefore, it is necessary to perform different checks of the analysis. After the extraction of angular distributions and finding the optical potential, it is useful to make a comparison of the model $\sigma^{D W B A}$ for different $R_{\text {cutoff }}$ parameters for the integration. The comparison should show that the selected optical potential is not much dependent on the nucleus interior. See left panel in Fig. 3, where the reaction $\mathrm{p}+{ }^{9} \mathrm{Be}$ was studied by A.M.Mukhamedzhanov et al. [14] using the reaction ${ }^{9} B\left({ }^{10} B,{ }^{10} B\right){ }^{9} \mathrm{Be}$ at Texas A\&M.

Since the single particle radial wave function $\varphi$ behavior depends quite strongly on the optical model potential, it is possible to inspect the radial behavior of $\varphi$ varying the potential parameter $r_{0}$. The tail should correspond to the Whitaker function. It should be visible, that the single particle wave function reaches the asymptotic form and can be replaced by the Whitaker function even at short distances, e.g. middle panel in Fig. 3 at $r>4.5 \mathrm{fm}$ [14].

Another important check is the test of dependence of $\mathrm{ANC}\left(C^{2}\right)$ on optical potential parameters. An example is given in Fig.3, right panel from A.M.Mukhamedzhanov et al. [14], where the ANC was plotted in dependence on SPANC $b$, as it represented a better way to determine the changes in $r_{0}$ 

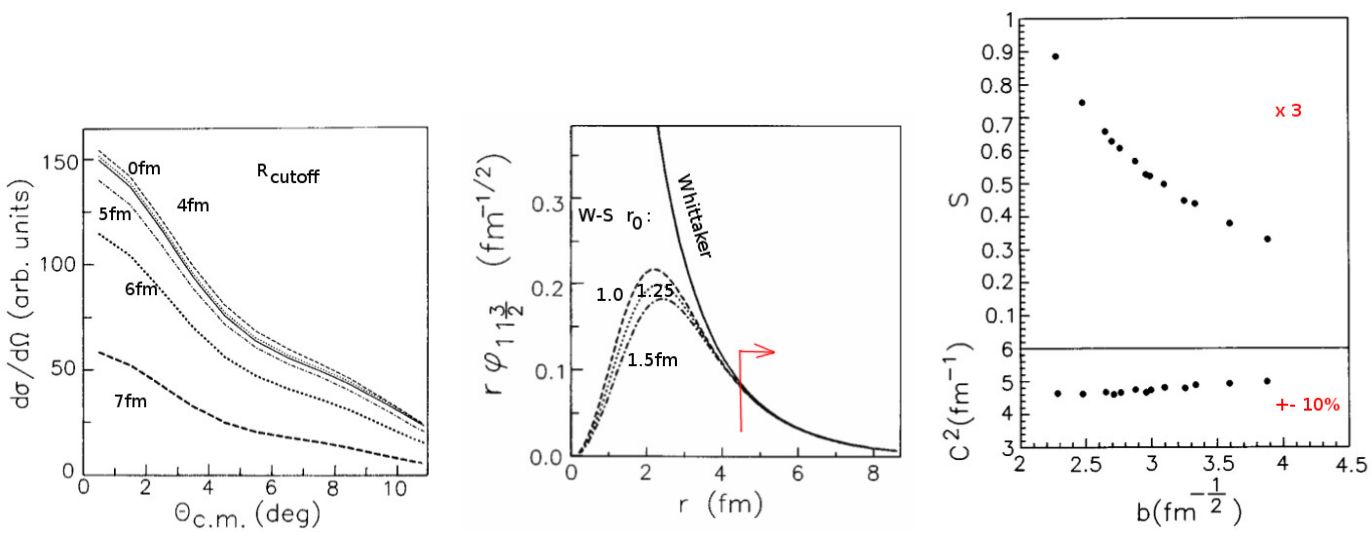

Figure 3. Left panel: The $R_{\text {cutoff }}$ parameter was varied from zero to $7 \mathrm{fm}$ and it is shown that the dependence of the $\sigma^{D W B A}$ is weak up to $4 \mathrm{fm}$ as done by A.M.Mukhamedzhanov et al. [14], Middle panel: The single particle radial wave function $\varphi$ variation inside the nucleus and the asymptotic above $r>4.5 \mathrm{fm}$, Right panel: ANC varies bellow $10 \%$ for range of different optical model parameters, for comparison a strong dependence of the SF is shown.

and $a$ parameters of the bound state potential. The variation of the ANC was there shown to be bellow $9 \%$, while for comparison, SF varied by a factor 3 for the same variation of $r_{0}, a$.

With the appearance of resonances near the threshold (or the reaction in study) region, the direct radiative capture is not the only nor the main process contributing to the $(p, \gamma)$ process. The resonant capture plays an important role and frequently its contribution is much more important. However, the direct capture can contribute in an indirect way. The coherent sum of the direct and indirect contributions can create important effects in the cross section (or astrophysical $\mathrm{S}$ factor) in a region of interest.

An instructive example is the ${ }^{15} \mathrm{~N}(\mathrm{p}, \gamma){ }^{16} \mathrm{O}$ reaction measured and analyzed by our group, A.M.Mukhamedzhanov et al. [15], where two $1^{-}$resonances (left panel of Fig.4) dominate the low energy reagion. The deduced ANC in [15] was $C^{2}=192 . \pm 26 \mathrm{fm}^{-1}\left(C=13.856 \mathrm{fm}^{-1 / 2}\right)$, the deduced astrophysical $\mathrm{S}$ factor was $S(0)=36.0(6.0) \mathrm{keVb}$, which was below the previous findings and this resulted in a change of the estimation of the leak from this CNO cycle reaction from one every 2200 (300) cycles to one per 1200, which is almost a factor two. This measurement was later confirmed by P.J.LeBlanc and LUNA collaboration [16], where the $S(0)$ was found $S(0)=39.6 \pm 2.6 \mathrm{keVb}$.

In response to [16], Mukhamedzhanov, La Cognata and Kroha [17] have discussed the treatment of ANC in the above work, which resulted in a new perspective of using ANCs as an auxiliary information to pinpoint spectroscopic factors.

For a comparison the right panel of Fig.4 shows the influence of different values of ANCs to the description of resonances.

\section{Summary}

The principles of the indirect method for determination of direct radiative capture - Asymptotic Normalization Coefficients method (ANC) - and it's evolution from spectroscopic factors were presented. To validate the approximations of the method, it is necessary to do specific checks with the model. Examples from real case analysis were presented. The importance of the knowledge of ANCs also 

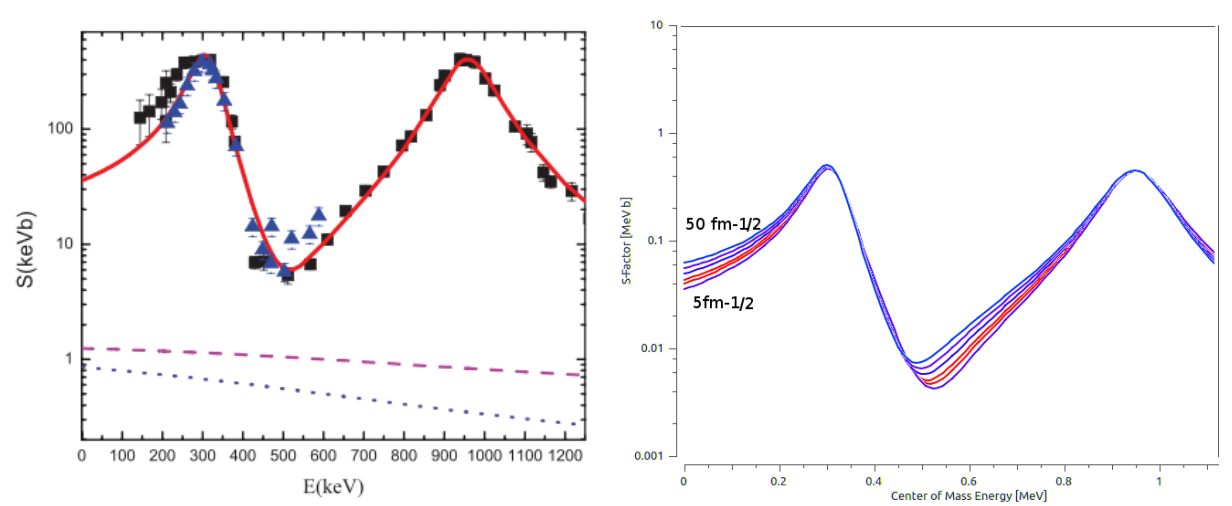

Figure 4. Left panel: Astrophysical S-factor based on R-matrix fit of the known data and the deduced ANC in A.M.Mukhamedzhanov et al. [15]. The dashed line is ANC for the ground state. Right panel: variation of ANC $\left(C_{\text {mean }}=13.85 \mathrm{fm}^{-1 / 2}\right.$ and $10 \mathrm{fm}^{-1 / 2}$ in red) entering the R-matrix fit shows the highest sensitivity at very low energy, which is a region for e.g. CNO scenario.

in resonant radiative capture was pointed out in the example. This publication was supported by OP RDE, MEYS, Czech Republic under the project SPIRAL2-CZ, CZ.02.1.01/0.0/0.0/16_013/0001679.

\section{References}

[1] E. Fermi, E. Amaldi, O. D’Agostino, F. Rasetti, E. Segre, Proceedings of the Royal Society of London A: Mathematical, Physical and Engineering Sciences 146, 483 (1934)

[2] G. Breit, E. Wigner, Phys. Rev. 49, 519 (1936)

[3] S.N. Ghoshal, Phys. Rev. 80, 939 (1950)

[4] S.T. Butler, Phys. Rev. 106, 272 (1957)

[5] S.T. Butler, Phys. Rev. 80, 1095 (1950)

[6] N.K. Glendenning, Direct Nuclear Reactions (ACADEMIC PRESS, INC., 1983)

[7] R. Furnstahl, H.W. Hammer, Physics Letters B 531, 203 (2002)

[8] B.K. Jennings, ArXiv e-prints (2011), 1102.3721

[9] A.M. Mukhamedzhanov, A.S. Kadyrov, Phys. Rev. C 82, 051601 (2010)

[10] X.D. Liu, M.A. Famiano, W.G. Lynch, M.B. Tsang, J.A. Tostevin, Phys. Rev. C 69, 064313 (2004)

[11] A.M. Mukhamedzhanov, N.K. Timofeyuk, JETP Lett 52, 282 (1990)

[12] L.D.Blokhintsev, Fiz.Elem.Chastits At.Yadra 8, 1189 (1977)

[13] L.D.Blokhintsev, Fiz.Elem.Chastits At.Yadra 15, 1296 (1984)

[14] A.M. Mukhamedzhanov, H.L. Clark, C.A. Gagliardi, Y.W. Lui, L. Trache, R.E. Tribble, H.M. Xu, X.G. Zhou, V. Burjan, J. Cejpek et al., Phys. Rev. C 56, 1302 (1997)

[15] A.M. Mukhamedzhanov, P. Bém, V. Burjan, C.A. Gagliardi, V.Z. Goldberg, Z. Hons, M. La Cognata, V. Kroha, J. Mrázek, J. Novák et al., Phys. Rev. C 78, 015804 (2008)

[16] P.J. LeBlanc, G. Imbriani, J. Görres, M. Junker, R. Azuma, M. Beard, D. Bemmerer, A. Best, C. Broggini, A. Caciolli et al., Phys. Rev. C 82, 055804 (2010)

[17] A.M. Mukhamedzhanov, M. La Cognata, V. Kroha, Phys. Rev. C 83, 044604 (2011) 